\title{
Meniscal Extrusion Progresses Shortly after the Medial Meniscus Posterior Root Tear
}

\author{
Takayuki Furumatsu, MD, Yuya Kodama, MD, Yusuke Kamatsuki, MD, Tomohito Hino, MD, \\ Yoshiki Okazaki, MD, and Toshifumi Ozaki, MD \\ Department of Orthopaedic Surgery, Okayama University Graduate School, Okayama, Japan
}

\begin{abstract}
Purpose: Medial meniscus posterior root tears (MMPRT) induce medial meniscus extrusion (MME). However, the time-dependent extent of MME in patients suffering from the MMPRT remains unclear. This study evaluated the extent of MME after painful popping events that occurred at the onset of the MMPRT.

Materials and Methods: Thirty-five patients who had an episode of posteromedial painful popping were investigated. All the patients were diagnosed as having an MMPRT by magnetic resonance imaging (MRI) within 12 months after painful popping. Medial meniscus body width (MMBW), absolute MME, and relative MME (100×absolute MME/MMBW) were assessed among three groups divided according to the time after painful popping events: early period ( $<1$ month), subacute period (1-3 months), and chronic period (4-12 months).

Results: In the early period, absolute and relative MMEs were $3.0 \mathrm{~mm}$ and $32.7 \%$, respectively. Absolute MME increased up to $4.2 \mathrm{~mm}$ and 5.8 $\mathrm{mm}$ during the subacute and chronic periods, respectively. Relative MME also progressed to $49.2 \%$ and $60.3 \%$ in the subacute and chronic periods, respectively.

Conclusions: This study demonstrated that absolute and relative MMEs increased progressively within the short period after the onset of symptomatic MMPRT. Our results suggest that early diagnosis of an MMPRT may be important to prevent progression of MME following the MMPRT.
\end{abstract}

Keywords: Meniscus, Tear, Root, Extrusion

\section{Introduction}

The posterior root of the medial meniscus (MM) can serve as an anchor to regulate the meniscal shift during the knee motion and load bearing. Injuries to the MM posterior root, including complete radial and/or oblique tears adjacent to the ligamentous insertion and posterior horn, lead to accelerated degeneration of the knee joint articular cartilage by disrupting meniscal func-

Received May 4, 2017; Revised June 6, 2017;

Accepted July 3, 2017

Correspondence to: Takayuki Furumatsu, MD

Department of Orthopaedic Surgery, Okayama University Graduate

School, 2-5-1 Shikatacho, Kitaku, Okayama 700-8558, Japan

Tel: +81-86-235-7273, Fax: +81-86-223-9727

E-mail: matino@md.okayama-u.ac.jp

This is an Open Access article distributed under the terms of the Creative Commons Attribution Non-Commercial License (http://creativecommons.org/licenses/by-nc/4.0/) which permits unrestricted non-commercial use, distribution, and reproduction in any medium, provided the original work is properly cited. tions $^{1)}$. In addition, the medial meniscus posterior root tear (MMPRT) leads to abnormal biomechanics of the tibiofemoral joint and the inability to convert axial loads into hoop stresses by inducing radial displacement of the $\mathrm{MM}$, also called medial meniscus extrusion (MME) ${ }^{2,3)}$. Extrusion (subluxation, bulging, and radial/extra-articular displacement) of the MM is associated with a loss of medial compartment cartilage volume ${ }^{4}$, medial joint space narrowing ${ }^{5)}$, severity of osteoarthritis as reflected by radiographic Kellgren-Lawrence grade ${ }^{6,7)}$, degenerative knee abnormalities ${ }^{8)}$, and knee joint pain ${ }^{9)}$ in patients with osteoarthritic knees. In addition, meniscal extrusion predicts an increase in subchondral bone lesions and tibial plateau expansion in osteoarthritic knees ${ }^{10)}$. MME is also a characteristic finding on magnetic resonance imaging (MRI) of patients having the MMPRT ${ }^{11)}$. However, the time-dependent extent of MME in patients suffering from the MMPRT remains unclear. Moreover, the relationship among the MME severity, treatment choice, and optimal timing for surgical intervention has not been elucidated. 
The MMPRT can occur especially in middle-aged or older patients who have a single event of posteromedial painful popping sensation during light activities such as using stairs and squatting ${ }^{12,13)}$. The positive predictive value of painful popping in identifying the MMPRT is $96.5 \%$ and the specificity is $99.5 \%{ }^{13)}$. In the treatment of the MMPRT, early diagnosis and appropriate surgical intervention are important to obtain a successful clinical outcome and prevent rapid progression of degenerative knee diseases ${ }^{1,14}$. MRI-based characteristic findings such as a radial tear, cleft/truncation, ghost, and giraffe neck signs provide high diagnostic accuracy and specificity ${ }^{11,15}$. However, we cannot obtain information about the exact onset of the MMPRT from these MRI findings. Although the sensitivity of a painful popping for the detection of an MMPRT is low $(35 \%)^{13)}$, the memory of hearing a painful popping sound seems to be necessary to determine the timing of the MMPRT onset and MRI examination and treatment strategy for the symptomatic MMPRT.

Repair of the MMPRT can reduce the mean tibiofemoral contact pressure by increasing the tibiofemoral contact area as demonstrated in a human cadaveric knee study ${ }^{16)}$. Several repair techniques such as transtibial pullout repair, suture anchor-dependent repair, direct all-inside repair, and posterior reattachment of the MM posterior root have been developed for arthroscopic treatment of the MMPRT ${ }^{1,14}$. MMPRT repairs produce more favorable clinical outcomes compared with conservative treatments ${ }^{17)}$. Despite the favorable clinical results of transtibial pullout repair of the MMPRT, several meta-analyses have demonstrated that many patients show no significant reduction in MME following the MMPRT repair ${ }^{18,19)}$. On the other hand, there are few reports that demonstrate the MMPRT repair can reduce MME. For example, MMPRT pullout repairs decreased MME from 3.13 $\mathrm{mm}$ to $2.94 \mathrm{~mm}^{20)}$. Patients with decreased MME following the MMPRT pullout repair had more favorable clinical scores than those with increased MME at 1 year postoperatively ${ }^{21}$. Based on these findings, a timely surgical intervention before causing a severe MME may be a critical factor for the treatment of the MMPRT and preventing progression of degenerative knee joint diseases. In this study, we evaluated the time-dependent changes in the extent of MME after painful popping events involved in the MMPRT onset.

\section{Materials and Methods}

\section{Study Materials}

This study received the approval of our Institutional Review Board, and written informed consent was obtained from all pa- tients. Thirty-five patients (32 females and 3 males) who had an episode of posteromedial painful popping between September 2013 and August 2016 were included (Table 1). All the patients were diagnosed as having the MMPRT with MRI examinations. The presence of an MMPRT was defined according to characteristic MRI findings (cleft/giraffe neck/radial tear signs of the MM posterior root within $9 \mathrm{~mm}$ from the attachment, ghost sign, and MM extrusion ${ }^{11,15)}$ ). Patients who had an MMPRT without a memory of painful popping $(\mathrm{n}=3)$ and an MMPRT with previous meniscal injury and/or knee surgery $(n=2)$ were excluded. The mean age of the patients was 63.5 years. Types of the MMPRT were determined by careful arthroscopic examinations (24 knees) or arthroplasty-associated direct observations (5 knees) according to the meniscal root tear classification (Table 1) ${ }^{22}$. Transtibial pullout repairs (16 knees) were performed in patients who met operative indications for the pullout repair ${ }^{23-25}$. All-inside meniscal repairs (6 knees) were performed in the other patients. There was no patient who underwent meniscectomy alone. Patients were examined by MRI scans prior to surgical treatments and within 12 months after painful popping events. Six patients were treated conservatively because of their low activities and hesitation about surgical treatments. Conservative treatments involved partial weight bearing and a stick, crutch, knee brace, and medical guidance were used appropriately.

\section{MRI-Based Measurements}

MRI evaluation was performed using an Achieva 1.5 T (Philips, Amsterdam, The Netherlands) with a knee coil. Standard

Table 1. Demographics and Clinical Characteristics

\begin{tabular}{lc}
\multicolumn{1}{c}{ Characteristic } & Value \\
\hline No. of patients & 35 \\
Sex (male:female) & $3: 32$ \\
Root tear classification (29 knees) & \\
Type 1 & 5 \\
Type 2 & 22 \\
Type 3 & 1 \\
Type 4 & 1 \\
Type 5 & 0 \\
Age (yr) & $63.5 \pm 10.3$ \\
Height $(\mathrm{m})$ & $1.55 \pm 0.06$ \\
Body weight $(\mathrm{kg})$ & $64.9 \pm 14.4$ \\
Body mass index $\left(\mathrm{kg} / \mathrm{m}^{2}\right)$ & $26.9 \pm 5.3$ \\
Femorotibial angle $\left({ }^{\circ}\right)$ & $177.5 \pm 1.6$ \\
\hline
\end{tabular}

Values are presented as mean \pm standard deviation or number. 
sequences included sagittal [repetition time (TR)/echo time (TE), 742/18], coronal (TR/TE, 637/18), and axial (TR/TE, 499/18) T2weighted fast-field echo with a $20^{\circ}$ flip angle. The slice thickness was $3 \mathrm{~mm}$ with a $0.6-\mathrm{mm}$ gap. The field of view was 16 (or 17) $\mathrm{cm}$ with an acquisition matrix size of $205 \times 256$ (or $200 \times 368)^{26-29)}$. The MRI-based medial meniscus body width (MMBW), absolute MME, and medial meniscus height (MMH) were assessed. The MMBW was measured from the inner border to the outer border of the MM on the coronal image that crossed the midpoint of the anteroposterior length of the MM. The absolute MME was measured from the medial margin of the tibial plateau to the outer border of the MM. The MMH was measured from the inferior margin to the superior margin of the MM on the same coronal image. The relative MME was calculated using the following formula: 100×absolute MME/MMBW (\%).

\section{Statistical Analysis}

Data were presented as means \pm standard deviations. Differences among groups were compared using the one-way analysis of variance (ANOVA) with Tukey post hoc tests. Power and statistical analyses were performed using EZR (Saitama Medical Center, Saitama, Japan), which is a graphical user interface for $\mathrm{R}$ (the $\mathrm{R}$ Foundation for statistical computing). Significance was set to $\mathrm{p}<0.05$. Two orthopedic surgeons ( $\mathrm{TH}$ and $\mathrm{YK}$ ) independently measured MRI-based values of the MM in a blinded manner. Each observer performed each measurement twice, at least 2 weeks apart. The reliability of the measurements was assessed by examining the inter-observer and intra-observer reliabilities with the intraclass correlation coefficient (ICC). An ICC values of $>0.80$ was considered to represent a reliable measurement. Linear regression analysis was used to assess the correlation between the absolute MME and the other factors (age, height, body mass index, and femorotibial angle). A good correlation was defined as $\mathrm{R}^{2} \geq 0.60$, fair correlation as $\mathrm{R}^{2} \geq 0.50$, and poor correlation as $\mathrm{R}^{2}<0.50$.

\section{Results}

On the MRI-based measurements of the MM, the MMBW showed no significant change among three groups divided according to the time after painful popping events into the early period ( $<1$ month), subacute period (1-3 months), and chronic period (4-12 months) (Table 2 and Fig. 1A). In the early period, the absolute and relative MMEs were $3.0 \pm 1.2 \mathrm{~mm}$ and $32.7 \% \pm 12.2 \%$, respectively (Table 2, Fig. $1 \mathrm{~B}$ and $\mathrm{C}$ ). However, the absolute MME significantly increased up to $4.2 \pm 1.2 \mathrm{~mm}$ and $5.8 \pm 1.6 \mathrm{~mm}$ in the subacute and chronic periods, respectively. The relative MME also progressed to $49.2 \% \pm 11.9 \%$ and $60.3 \% \pm 14.9 \%$ in the subacute and chronic periods, respectively. Significant difference in the MMH was observed between the early and chronic durations ( $p=0.014)$ (Table 2 and Fig. 1D). The inter-observer and

Table 2. Changes in the MMBW, MME, and MMH after Painful Popping Events

\begin{tabular}{lccc}
\hline \multicolumn{1}{c}{ Variable } & $\begin{array}{c}\text { Early }(\mathrm{n}=15) \\
<1 \text { month }\end{array}$ & $\begin{array}{c}\text { Subacute }(\mathrm{n}=17) \\
1-3 \text { months }\end{array}$ & $\begin{array}{c}\text { Chronic }(\mathrm{n}=17) \\
4-12 \text { months }\end{array}$ \\
\hline MMBW $(\mathrm{mm})$ & $9.1 \pm 1.2$ & $8.5 \pm 1.1$ & $9.6 \pm 1.3$ \\
Absolute MME $(\mathrm{mm})^{\mathrm{a})}$ & $3.0 \pm 1.2$ & $4.2 \pm 1.2$ & $5.8 \pm 1.6$ \\
Relative MME $(\%)^{\mathrm{a})}$ & $32.7 \pm 12.2$ & $49.2 \pm 11.9$ & $60.3 \pm 14.9$ \\
MMH $(\mathrm{mm})^{\mathrm{a})}$ & $6.8 \pm 1.7$ & $7.1 \pm 1.4$ & $8.4 \pm 1.6$ \\
\hline
\end{tabular}

Values are presented as mean \pm standard deviation.

MMBW: medial meniscus body width, MME: medial meniscus extrusion, MMH: medial meniscus height.

a) Significant differences were observed among groups.
A

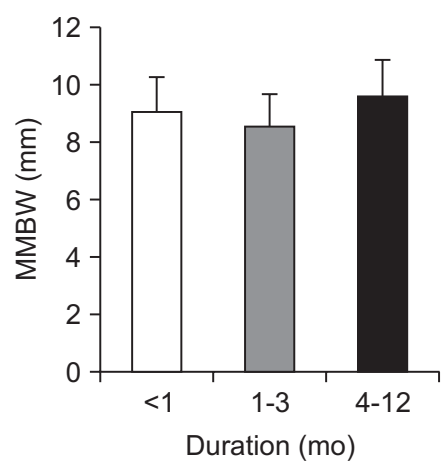

B

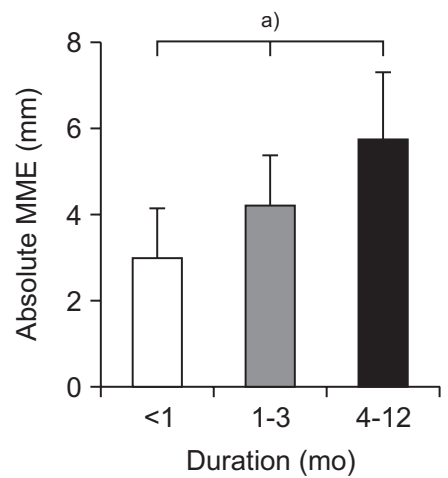

C

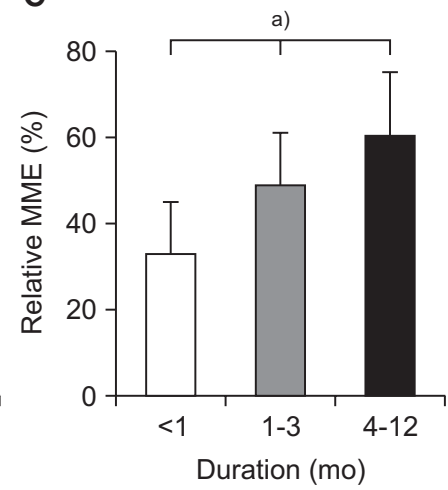

D

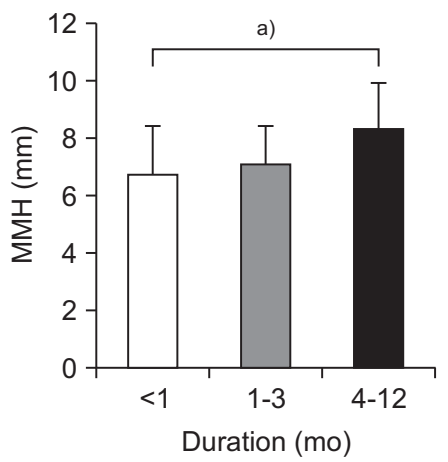

Fig. 1. Magnetic resonance imaging-based measurements of the medial meniscus after the onset of medial meniscus posterior root tears. (A) Medial meniscus body width (MMBW). (B) Absolute medial meniscus extrusion (MME). (C) Relative MME (100×absolute MME/MMBW). (D) Medial meniscus height $(\mathrm{MMH}) .{ }^{\mathrm{a})} \mathrm{p}<0.05$. 
intra-observer reliabilities for the measurements were considered satisfactory (mean ICC values $>0.94$ ).

A poor correlation was observed between the absolute MME and age $\left(\mathrm{R}^{2}=0.125\right)$. The absolute MME and the other factors (height, body mass index, and tibiofemoral angle) also showed poor correlations $\left(\mathrm{R}^{2}=0.009,0.011\right.$, and 0.001 , respectively).

Representative sequential MRI scans showed a post-traumatic progression of MME after a painful popping event of the knee (Fig. 2). A 65-year-old female had a single event of painful popping of her right knee in the full squatting position. Minor MME $(1.3 \mathrm{~mm})$ and specific MRI findings of the MMPRT were ob- served at 1 week after the painful popping event (Fig. 2A). The MME increased to $5.0 \mathrm{~mm}$ at 10 weeks after the MMPRT (Fig. 2B). The MME progressed to $5.7 \mathrm{~mm}$ at 18 weeks post-MMPRT (Fig. 2C). Bone edematous change in the medial femoral condyle was detected on MRI at 10 and 18 weeks after the MMPRT onset (Fig. 2B and C). Radiographic findings associated with spontaneous osteonecrosis of the knee progressed to the Koshino classification stage 3 (collapsed stage) at 18 weeks (Fig. 3A) ${ }^{30)}$. She underwent arthroscopic assessment and unicompartmental knee arthroplasty of her right knee at 20 weeks after the painful popping because of severe knee pain (Fig. 3B and C).
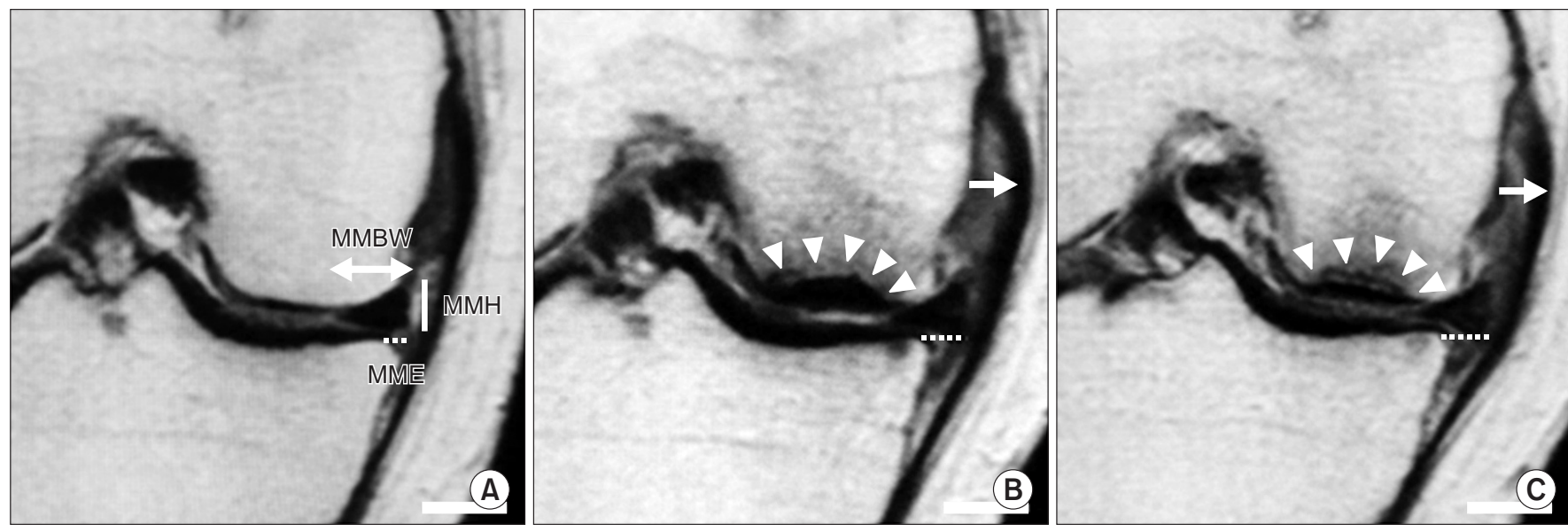

Fig. 2. Medial meniscus extrusion (MME) increased after the medial meniscus posterior root tear (MMPRT) onset in a 65-year-old female (the right knee). (A) The coronal view showed minor MME 1week after a painful popping event indicating the MMPRT onset. Double-headed arrow: medial meniscus body width (MMBW). Dotted line: absolute MME. Solid line: medial meniscus height (MMH). (B) The MME increased 10 weeks after the MMPRT. Note the bone edema of the medial femoral condyle (arrowheads) and medial shift of the medial collateral ligament (arrow). (C) The MME progressed 18 weeks after the MMPRT. Bars: $1 \mathrm{~cm}$.
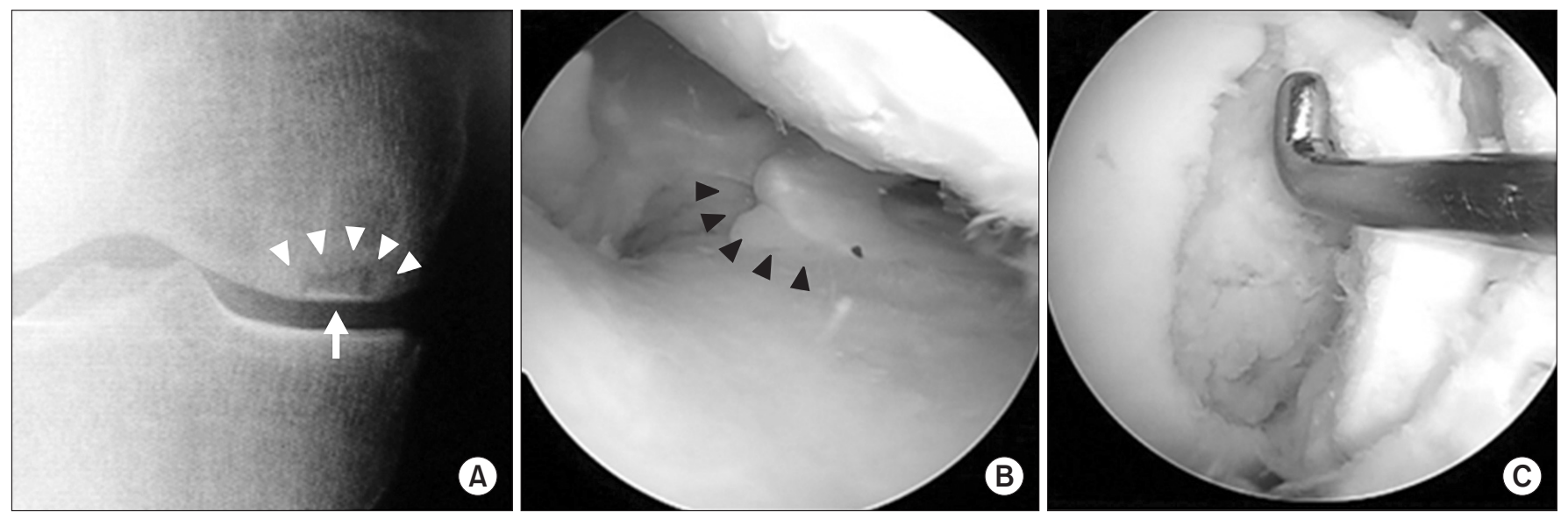

Fig. 3. Spontaneous osteonecrosis of the knee following the medial meniscus posterior root tear (MMPRT) in the 65-year-old female shown in Fig. 2 (the right knee). (A) Sclerotic halo (arrowheads) and calcified plate (arrow) were observed in a radiograph at 18 weeks after a painful popping event. Koshino classification: stage 3. (B) Arthroscopic findings at 20 weeks post-MMPRT. Arrowheads: type 2 MMPRT. (C) An unstable osteochondral flap. 


\section{Discussion}

The most important finding in this study was that the absolute MME increased progressively within 12 months after the onset of symptomatic MMPRT (Table 2 and Fig. 1B). The relative MME also increased during non-operative treatment period following the posteromedial painful popping event (Table 2 and Fig. 1C). These results may provide us with an important evaluation criterion for the MMPRT to determine the timing of surgical treatments. MME of $\geq 3 \mathrm{~mm}$ is more frequent in painful osteoarthritic knees than in contralateral painless knees, and more frequent in osteoarthritic knees that have a higher radiographic grade (Kellgren-Lawrence grade III) ${ }^{9}$. A radially displaced MM forms a bulged (or swelled) meniscal shape on the coronal MRI during the progression of osteoarthritic knees ${ }^{31}$. In addition, the status of MME can affect postoperative clinical outcome of the MMPRT transtibial pullout repair ${ }^{21)}$. Patients with decreased MME $(3.5 \pm 1.4$ $\mathrm{mm})$ at 1 year post-MMPRT pullout repairs have more favorable clinical outcomes and radiographic findings at 5 -year follow-up than those with increased MME $(5.1 \pm 1.4 \mathrm{~mm})$ at 1 year postoperatively ${ }^{21)}$. We consider that pullout repair-dependent positional restoration of the MM may be disturbed by a bulged/swelled $\mathrm{MM}$ and knee joint space narrowing concomitant with sequential MME increase after the MMPRT onset. Our study demonstrated that the mean absolute MME was $3.0 \pm 1.2 \mathrm{~mm}$ even in the early period less than 1 month after the MMPRT onset. Based on these findings, it seems that the symptomatic MMPRT should be treated with arthroscopic meniscal repair techniques as soon as possible following the diagnosis of the MMPRT if the patients meet surgical indications for the MMPRT repair.

Non-operative treatment of the MMPRT was associated with poor clinical outcome, worsening osteoarthritis of the knee, and a relatively high rate (16/52 knees, $31 \%)$ of total knee arthroplasty at a mean of 30 months after diagnosis of the MMPRT ${ }^{29)}$. The overall failure rate based on clinical and radiographic criteria was $87 \%$ in this literature. In addition, female gender was related to a higher rate of arthroplasty ${ }^{32}$. In biomechanical studies, the MMPRT led to excessive contact pressure on the articular surface of the knee by reducing the contact area of the knee joint cartilage $e^{2,3)}$. We consider that the MMPRT can suddenly deteriorate the status of articular cartilage and subchondral bone by altering the knee joint kinematics and homeostasis immediately. Therefore, the MMPRT-related osteonecrotic lesions and spontaneous osteonecrosis of the knee may be associated with female gender having osteoporotic subchondral bone quality.

Many studies have reported that MME is associated with pro- gression of symptomatic knee osteoarthritis ${ }^{4,699}$. Meniscus-tofemoral condyle congruity is essential for the development of circumferential hoop stresses and meniscal function. Abnormalities in the position of the MM and its coverage, such as the MMPRT, MME, and meniscectomy-related meniscal defects, can alter knee joint congruity and are associated with the progression of tibiofemoral osteoarthritis and cartilage degradation ${ }^{4)}$. Sung et al. ${ }^{33)}$ reported that the mean absolute MME and relative MME were $4.1 \pm 0.7 \mathrm{~mm}$ and $46.1 \% \pm 9.0 \%$ in 36 knees showing the MMPRT at a mean of 5.3-months symptom duration, respectively. In addition, MRI-based osteonecrotic lesions were observed in 12/36 knees (33\%) of the MMPRT patients ${ }^{33)}$. In our study, the absolute MME significantly increased from $3.0 \pm 1.2 \mathrm{~mm}$ to $5.8 \pm 1.6 \mathrm{~mm}$ post-MMPRT (Table 2 and Fig. 1). In the subacute period (1-3 months after the painful popping), the absolute and relative MME progressed to $4.2 \pm 1.2 \mathrm{~mm}$ and $49.2 \% \pm 11.9 \%$, respectively (Table 2). Based on these findings, the MMPRT should be treated within the subacute period after the MMPRT onset for preventing a substantial MME of more than $4 \mathrm{~mm}$. We consider that surgical intervention at an adequate timing may be important to prevent degenerative knee joint disease following the MMPRT if the patient meets operative indications. Further investigations will be required to determine the precise timing of operative treatment for the MMPRT.

There are several limitations in this study. MRI examinations were performed differently in terms of the number of examinations and duration from the onset of MMPRT to MRI assessment. Follow-up MRI scans were not performed in all the patients after the primary MRI-based diagnosis of the MMPRT. Repeated MRI examinations in the same patients will be needed to precisely assess the time-dependent MME progression following the MMPRT. In this study, we evaluated the MRI-based MME in a single knee flexion angle $\left(10^{\circ}\right)$ under non-weight bearing condition. Open MRI assessments of meniscal movement using thin slices in several knee flexion angles under loading condition will be required to enhance the diagnostic value of MME in the MMPRT treatment. In addition, three-dimensional reconstruction of the MM using dynamic MRI may be useful to understand the MME increase after the MMPRT onset. Our study was a retrospective comparative study with a small sample size. Additional follow-up MRI studies involving in a larger sample size will be required to evaluate the real effect of MME on the progression of post-MMPRT symptoms. 


\section{Conclusions}

This study demonstrated that the absolute and relative MME increased progressively within the short period after the onset of symptomatic MMPRT. Our results suggest that the accurate diagnosis of the MMPRT in the early period after the onset may be important to prevent the increase of MME following the MMPRT.

\section{Conflict of Interest}

No potential conflict of interest relevant to this article was reported.

\section{Acknowledgements}

We thank Drs. Shinichi Miyazawa, Takaaki Tanaka, and Hiroto Inoue for their clinical supports.

\section{References}

1. Bhatia S, LaPrade CM, Ellman MB, LaPrade RF. Meniscal root tears: significance, diagnosis, and treatment. Am J Sports Med. 2014;42:3016-30.

2. Padalecki JR, Jansson KS, Smith SD, Dornan GJ, Pierce CM, Wijdicks CA, Laprade RF. Biomechanical consequences of a complete radial tear adjacent to the medial meniscus posterior root attachment site: in situ pull-out repair restores derangement of joint mechanics. Am J Sports Med. 2014;42: 699-707.

3. Allaire R, Muriuki M, Gilbertson L, Harner CD. Biomechanical consequences of a tear of the posterior root of the medial meniscus: similar to total meniscectomy. J Bone Joint Surg Am. 2008;90:1922-31.

4. Berthiaume MJ, Raynauld JP, Martel-Pelletier J, Labonte F, Beaudoin G, Bloch DA, Choquette D, Haraoui B, Altman RD, Hochberg M, Meyer JM, Cline GA, Pelletier JP. Meniscal tear and extrusion are strongly associated with progression of symptomatic knee osteoarthritis as assessed by quantitative magnetic resonance imaging. Ann Rheum Dis. 2005; 64:556-63.

5. Bloecker K, Guermazi A, Wirth W, Benichou O, Kwoh CK, Hunter DJ, Englund M, Resch H, Eckstein F; OAI investigators. Tibial coverage, meniscus position, size and damage in knees discordant for joint space narrowing: data from the Osteoarthritis Initiative. Osteoarthritis Cartilage. 2013;21:
419-27.

6. Lee DH, Lee BS, Kim JM, Yang KS, Cha EJ, Park JH, Bin SI. Predictors of degenerative medial meniscus extrusion: radial component and knee osteoarthritis. Knee Surg Sports Traumatol Arthrosc. 2011;19:222-9.

7. Kawaguchi K, Enokida M, Otsuki R, Teshima R. Ultrasonographic evaluation of medial radial displacement of the medial meniscus in knee osteoarthritis. Arthritis Rheum. 2012; 64:173-80.

8. Stehling C, Souza RB, Hellio Le Graverand MP, Wyman BT, Li X, Majumdar S, Link TM. Loading of the knee during 3.0T MRI is associated with significantly increased medial meniscus extrusion in mild and moderate osteoarthritis. Eur J Radiol. 2012;81:1839-45.

9. Wenger A, Englund M, Wirth W, Hudelmaier M, Kwoh K, Eckstein F; OAI Investigators. Relationship of 3D meniscal morphology and position with knee pain in subjects with knee osteoarthritis: a pilot study. Eur Radiol. 2012;22:211-20.

10. Wang Y, Wluka AE, Pelletier JP, Martel-Pelletier J, Abram F, Ding C, Cicuttini FM. Meniscal extrusion predicts increases in subchondral bone marrow lesions and bone cysts and expansion of subchondral bone in osteoarthritic knees. Rheumatology (Oxford). 2010;49:997-1004.

11. Choi SH, Bae S, Ji SK, Chang MJ. The MRI findings of meniscal root tear of the medial meniscus: emphasis on coronal, sagittal and axial images. Knee Surg Sports Traumatol Arthrosc. 2012;20:2098-103.

12. Han SB, Shetty GM, Lee DH, Chae DJ, Seo SS, Wang KH, Yoo $\mathrm{SH}$, Nha KW. Unfavorable results of partial meniscectomy for complete posterior medial meniscus root tear with early osteoarthritis: a 5- to 8-year follow-up study. Arthroscopy. 2010;26:1326-32.

13. Bae JH, Paik NH, Park GW, Yoon JR, Chae DJ, Kwon JH, Kim JI, Nha KW. Predictive value of painful popping for a posterior root tear of the medial meniscus in middle-aged to older Asian patients. Arthroscopy. 2013;29:545-9.

14. Bonasia DE, Pellegrino P, D'Amelio A, Cottino U, Rossi R. Meniscal root tear repair: why, when and how? Orthop Rev (Pavia). 2015;7:5792.

15. Furumatsu T, Fujii M, Kodama Y, Ozaki T. A giraffe neck sign of the medial meniscus: a characteristic finding of the medial meniscus posterior root tear on magnetic resonance imaging. J Orthop Sci. 2017;22:731-6.

16. LaPrade CM, Foad A, Smith SD, Turnbull TL, Dornan GJ, Engebretsen L, Wijdicks CA, LaPrade RF. Biomechanical consequences of a nonanatomic posterior medial meniscal 
root repair. Am J Sports Med. 2015;43:912-20.

17. LaPrade RF, LaPrade CM, James EW. Recent advances in posterior meniscal root repair techniques. J Am Acad Orthop Surg. 2015;23:71-6.

18. Feucht MJ, Kühle J, Bode G, Mehl J, Schmal H, Südkamp NP, Niemeyer P. Arthroscopic transtibial pullout repair for posterior medial meniscus root tears: a systematic review of clinical, radiographic, and second-look arthroscopic results. Arthroscopy. 2015;31:1808-16.

19. Chung KS, Ha JK, Ra HJ, Kim JG. A meta-analysis of clinical and radiographic outcomes of posterior horn medial meniscus root repairs. Knee Surg Sports Traumatol Arthrosc. 2016;24:1455-68.

20. Kim SB, Ha JK, Lee SW, Kim DW, Shim JC, Kim JG, Lee MY. Medial meniscus root tear refixation: comparison of clinical, radiologic, and arthroscopic findings with medial meniscectomy. Arthroscopy. 2011;27:346-54.

21. Chung KS, Ha JK, Ra HJ, Nam GW, Kim JG. Pullout fixation of posterior medial meniscus root tears: correlation between meniscus extrusion and midterm clinical results. Am J Sports Med. 2017;45:42-9.

22. LaPrade CM, James EW, Cram TR, Feagin JA, Engebretsen L, LaPrade RF. Meniscal root tears: a classification system based on tear morphology. Am J Sports Med. 2015;43:363-9.

23. Kodama Y, Furumatsu T, Fujii M, Tanaka T, Miyazawa S, Ozaki T. Pullout repair of a medial meniscus posterior root tear using a FasT-Fix ${ }^{\circledR}$ all-inside suture technique. Orthop Traumatol Surg Res. 2016;102:951-4.

24. Furumatsu T, Kodama Y, Fujii M, Tanaka T, Hino T, Kamatsuki Y, Yamada K, Miyazawa S, Ozaki T. A new aiming guide can create the tibial tunnel at favorable position in transtibial pullout repair for the medial meniscus posterior root tear. Orthop Traumatol Surg Res. 2017;103:367-71.

25. Fujii M, Furumatsu T, Kodama Y, Miyazawa S, Hino T, Kamatsuki Y, Yamada K, Ozaki T. A novel suture technique using the FasT-Fix combined with Ultrabraid for pullout repair of the medial meniscus posterior root tear. Eur J Orthop Surg Traumatol. 2017;27:559-62.
26. Furumatsu T, Miyazawa S, Tanaka T, Okada Y, Fujii M, Ozaki T. Postoperative change in medial meniscal length in concurrent all-inside meniscus repair with anterior cruciate ligament reconstruction. Int Orthop. 2014;38:1393-9.

27. Fujii M, Furumatsu T, Miyazawa S, Okada Y, Tanaka T, Ozaki T, Abe N. Intercondylar notch size influences cyclops formation after anterior cruciate ligament reconstruction. Knee Surg Sports Traumatol Arthrosc. 2015;23:1092-9.

28. Narazaki S, Furumatsu T, Tanaka T, Fujii M, Miyazawa S, Inoue $\mathrm{H}$, Shimamura Y, Saiga K, Ozaki T. Postoperative change in the length and extrusion of the medial meniscus after anterior cruciate ligament reconstruction. Int Orthop. 2015;39:2481-7.

29. Kashihara N, Furumatsu T, Kodama Y, Tanaka T, Ozaki T. Concurrent lateral meniscal repair with anterior cruciate ligament reconstruction induces the extrusion of the lateral meniscus: assessments of magnetic resonance images. Acta Med Okayama. 2016;70:441-8.

30. Koshino T. The treatment of spontaneous osteonecrosis of the knee by high tibial osteotomy with and without bonegrafting or drilling of the lesion. J Bone Joint Surg Am. 1982; 64:47-58.

31. Wenger A, Wirth W, Hudelmaier M, Noebauer-Huhmann I, Trattnig S, Bloecker K, Frobell RB, Kwoh CK, Eckstein F, Englund M. Meniscus body position, size, and shape in persons with and persons without radiographic knee osteoarthritis: quantitative analyses of knee magnetic resonance images from the osteoarthritis initiative. Arthritis Rheum. 2013;65: 1804-11.

32. Krych AJ, Reardon PJ, Johnson NR, Mohan R, Peter L, Levy BA, Stuart MJ. Non-operative management of medial meniscus posterior horn root tears is associated with worsening arthritis and poor clinical outcome at 5-year follow-up. Knee Surg Sports Traumatol Arthrosc. 2017;25:383-9.

33. Sung JH, Ha JK, Lee DW, Seo WY, Kim JG. Meniscal extrusion and spontaneous osteonecrosis with root tear of medial meniscus: comparison with horizontal tear. Arthroscopy. 2013;29:726-32. 